\title{
A Comprehensive Lighting Configuration for Efficient Indoor Visible Light Communication Networks
}

\author{
Thai-Chien Bui, ${ }^{1}$ Suwit Kiravittaya, ${ }^{1}$ Keattisak Sripimanwat, ${ }^{2}$ and Nam-Hoang Nguyen ${ }^{3}$ \\ ${ }^{1}$ Advanced Optical Technology (AOT) Laboratory, Department of Electrical and Computer Engineering, \\ Faculty of Engineering, Naresuan University, Muang, Phitsanulok, Thailand \\ ${ }^{2}$ ECTI Association, Klong Luang, Pathumthani, Thailand \\ ${ }^{3}$ Faculty of Electronics and Telecommunications, VNU University of Engineering and Technology, Hanoi, Vietnam
}

Correspondence should be addressed to Suwit Kiravittaya; suwitki@gmail.com

Received 8 September 2016; Accepted 17 October 2016

Academic Editor: Chen Chen

Copyright (C) 2016 Thai-Chien Bui et al. This is an open access article distributed under the Creative Commons Attribution License, which permits unrestricted use, distribution, and reproduction in any medium, provided the original work is properly cited.

\begin{abstract}
Design of an efficient indoor visible light communication (VLC) system requires careful considerations on both illumination and communication aspects. Besides fundamental factors such as received power and signal-to-noise ratio (SNR) level, studies on mobility scenarios and link switching process must be done in order to achieve good communication link quality in such systems. In this paper, a comprehensive lighting configuration for efficient indoor VLC systems for supporting mobility and link switching with constraint on illumination, received power, and SNR is proposed. Full connectivity in mobility scenarios is required to make the system more practical. However, different from other literatures, our work highlights the significance of recognizing the main influences of field of view angle on the connectivity performance in the practical indoor scenarios. A flexible link switching initiation algorithm based on the consideration of relative received power with adaptive hysteresis margin is demonstrated. In this regard, we investigate the effect of the overlap area between two light sources with respect to the point view of the receiver on the link switching performance. The simulation results show that an indoor VLC system with sufficient illumination level and high communication link quality as well as full mobility and support link switching can be achieved using our approach.
\end{abstract}

\section{Introduction}

Radio frequency is facing more difficulty to meet the rapidly growing demand for high data rate wireless transmission and ubiquitous network connectivity in indoor environment due to limited bandwidth and electromagnetic interference. Several next generation indoor wireless communications have been proposed recently [1]. In this regard, visible light communication (VLC) has been emerging to be a potential alternative and complementary technology to its radio frequency counterpart along with the recent advanced development of light emitting diode (LED) [2-4]. By using LEDs for dual-function of illumination and communication, VLC inherits the advantages of LED such as lower power consumption, longer lifetime, smaller size, and cooler operation. As a result, the physical layer of VLC has attracted much attention to researchers over the past few years that are focusing primarily on point-to-point communication for high data rate $[4,5]$. However, how to configure those high speed point-to-point systems into real scenarios is remaining as a difficulty especially for indoor environment. It is due to the stringent requirement of illumination standard, communication performance, and mobility.

A typical indoor VLC system requires multiple LEDs with wide half power angle $\Phi_{1 / 2}$ which is mounted on the room ceiling in order to meet the illumination requirement and support mobility with continuous data transmission. However, with respect of communication performance, such VLC system suffers from intersymbol interference (ISI) which is caused by multipath propagation at high data rate [6]. In general, high level of ISI limits the achievable data rate and reduces the quality of received data by decreasing the level of signal-to-noise ratio (SNR). One can design an optimal VLC system using less number of LEDs but placing at reasonable 
positions as well as reducing the field-of-view (FOV) angle $\Psi_{c}$ of receiver to optimize SNR level while still satisfying the illumination requirement and other constraints, that is, sufficient received power and power consumptions [7, 8]. Nevertheless, apart from the illumination level and link quality which is normally determined by SNR and received power level, connectivity and link switching performance are necessary in evaluating an indoor VLC system.

Connectivity is vitally important for mobility scenarios as it defines the communication coverage of the system viewing by FOV angle of the receiver in the receiver plane. The work in [9] demonstrated the influence of the luminance uniformity of the system on mobility. The other work in [10] proposed an angle diversity receiver to offer full mobility within a typical home with an area of $5 \times 5 \mathrm{~m}^{2}$. Obviously, full-connectivity is desired for any system since it helps continuous data connection in all the places in the room. However, improper position of LEDs as well as too small FOV angle at receivers often introduces blind spots and limits the connectivity performance in indoor VLC system. Moreover, changing the receiver plane leads to varying the connectivity as well since it changes the lighting area of an LED and view area of receiver. Hence, connectivity can be adjusted within a range of receiver plane and FOV angle by simply designing the lighting layout.

Link switching in VLC is also an important issue as it is understood as the "handover process" in cellular wireless communication. It is thus considered as an indispensable process to maintain and improve the communication link in the scenario of mobility or interference in multi-LED VLC system [11]. In the literature, techniques for vertical handover between VLC hot spot and RF base station have been proposed in many works such as $[12,13]$. However, horizontal handover when user would like to switch from receiving information of one light source to the other has not attracted much attention. The work in [14] demonstrated the benefit of applying handover algorithm on the link in terms of bandwidth usage and data transmission rate. A novel prescanning and received signal strength (RSS) prediction technique is proposed to reduce link switching delay and unnecessary link switching ratio [15]. In designing the system, demonstrating the influence of different parameters of indoor VLC system on a link switching algorithm is required. To do this, the parameters related to the overlap area between LEDs are taken into account since switching process occurred in this area. As in cellular wireless systems, different algorithms used require different overlap area conditions. However, in general, this overlap area should be large enough for satisfying the link switching initiation conditions and seamless connectivity while switching process occurred, meaning that this overlap area depends on the initiation algorithm used and the link switching delay time.

In this paper, we design indoor VLC system by studying the effect of setting lighting positions and setting FOV angle on connectivity and link switching performance as well as illumination and link quality. The paper is structured as follows: In Section 2, the system model used in the present method and its basic performances are shown. In Section 3, the details of the connectivity and link switching conditions

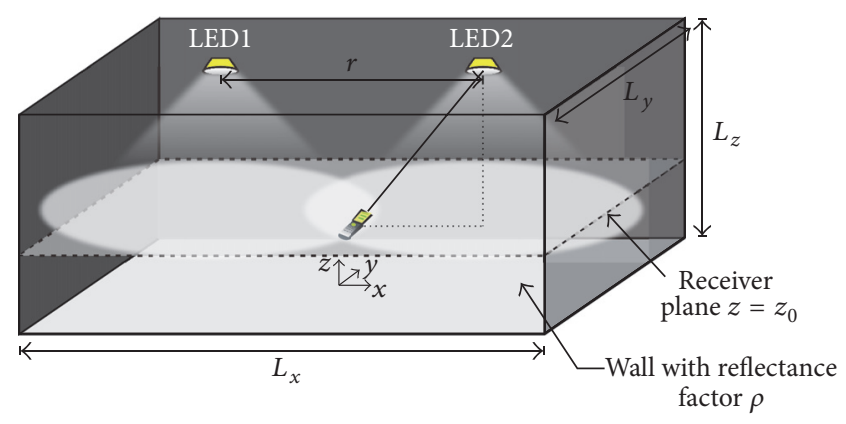

FIGURE 1: An indoor VLC system model with two LEDs tiled in the ceiling of a room dimension of $L_{x} \times L_{y} \times L_{z}$ (length, width, height).

TABLE 1: Simulation domain parameters.

\begin{tabular}{lc}
\hline Parameters and symbols & Values (unit) \\
\hline Room size $L_{x} \times L_{y} \times L_{z}$ & $5(\mathrm{~m}) \times 2.5(\mathrm{~m}) \times 2.5(\mathrm{~m})$ \\
Distance between LEDs $r$ & $2.5(\mathrm{~m})$ \\
Receiver plane $z_{0}$ & $0.85(\mathrm{~m})$ \\
Half-power angle $\Phi_{1 / 2}$ & $70\left(^{\circ}\right)$ \\
Transmitted optical power $P_{t}$ & $72(\mathrm{~W})$ \\
Center luminous intensity $I_{0}$ & $2628(\mathrm{~cd})$ \\
Reflectance factor of the walls $\rho$ & 0.8 \\
\hline
\end{tabular}

are presented. In Section 4, simulation results to evaluate the performance of the configuration method are presented and discussed. Finally, Section 5 concludes the work.

\section{System Model and Basic Performance}

In a conventional indoor VLC, a regular and alignment distribution of LEDs is normally used since it gives uniform lighting, uniform overlap areas, and design simplicity. Moreover, multiple-input multiple-output (MIMO) system can be realized with this LED configuration [16]. Consequently, demonstrating on the system with only two LEDs can be easily generalized to such MIMO systems with multiple LEDs. We therefore further simplify our VLC system model by considering only two LEDs installed on the ceiling of a room size of $L_{x} \times L_{y} \times L_{z}$ (length, width, height) as shown in Figure 1. The distance between the two LEDs is denoted as $r$. Each LED has a half-power angle $\Phi_{1 / 2}$, transmitted power $P_{t}$, and center luminous intensity $I_{0}$. It is assumed that VLC's receiver is a mobile terminal (MT) that can move on a plane $z=z_{0}$ above the floor. The reflectance factor of the wall is $\rho$. For later numerical simulation, all above parameters are set to typical values and summarized in Table 1 .

2.1. Illumination Distribution with First Reflection. Illumination requirement should be considered as the first priority in indoor VLC, as it is the primary functionality of the system. In common indoor environment, illumination criterion is standardized by ISO (International Organization for Standardization). Referring to this set of standards, lightings for an indoor office workspace integrated VLC should be 
designed in the way so that illumination level between 300 and $1500 \mathrm{~lx}$ is achieved in all the places in the room [17]. To calculate illumination for a VLC system, it is assumed that each LED has a Lambertian radiation pattern, with the Lambert index $m$, depending on the half-power angle of LED $\Phi_{1 / 2}$ as $m=-1 / \log _{2}\left(\cos \Phi_{1 / 2}\right)$.

The line-of-sight (LOS) horizontal illumination $E_{\text {hor }}$ at a point depends on Lambert index $m$, the angle of incidence $\psi_{d}$, the angle of irradiance $\phi_{d}$, the center luminous intensity $I_{0}$, and the distance between LED and receiver's surface $D_{d}$ [18]. In this paper, we make an assumption that the detector's surface is always in the vertical direction perpendicular to the plane of the ceiling. It means that for LOS link the angle of incidence $\psi_{d}$ is always identical with the angle of irradiance $\phi_{d}$. Hence, the formula of LOS horizontal illumination $E_{\text {hor }}$ can be rewritten as

$$
E_{\text {hor }}=I_{0} \frac{\cos ^{m+1}\left(\psi_{d}\right)}{D_{d}^{2}}
$$

If one considers light reflection at walls, the total illumination $E_{\text {total }}$, from the directed light and first reflection at a point, is given by $E_{\text {total }}=E_{\text {hor }}+E_{\text {ref }}$, where $E_{\text {ref }}$ is the illumination level from reflections. For simplicity, only the first reflection from the wall is considered. In this case, the angle of irradiance $\phi_{r}$ is different from the angle of incidence $\psi_{r}$ and $E_{\text {ref }}$ can be calculated as follows [19]:

$$
\begin{aligned}
& E_{\text {ref }} \\
& \qquad \int_{\text {walls }} I_{0} \frac{(m+1)}{2 \pi^{2}} \frac{\rho \cos ^{m} \phi_{r} \cos \psi_{r} \cos \alpha \cos \beta}{D_{1}^{2} D_{2}^{2}} d A_{\text {wall }},
\end{aligned}
$$

where $d A_{\text {wall }}$ is the reflective area of small region on the wall, $\alpha$ is the angle of irradiance to a reflective point, $\beta$ is the angle of irradiance to the receiver, $\psi_{r}$ is the angle of incidence, $D_{1}$ is the distance between the LED and the reflective point, and
$D_{2}$ is the distance between the reflective point to the receiver. The schematic illustration of both LOS link and first reflection link is shown in Figure 2.

2.2. Received Power and SNR. Appropriated received power level is desired in any communication system, yet it normally comes with the penalty of power consumption or interference. In VLC, apart from increasing transmitted power and number of LEDs used which are known as normal ways to increase received power, there are still other ways such as adjusting the placement of LEDs in a proper way or decreasing the field of view of receiver. To calculate received power, the study of the channel is required. In an optical link, the channel DC gain on directed path is given as

$$
\begin{aligned}
& H(0) \\
& = \begin{cases}\frac{(m+1) A}{2 \pi D_{d}^{2}} \cos ^{m+1} \psi_{d} T_{s}\left(\psi_{d}\right) g\left(\psi_{d}\right), & 0 \leq \psi_{d} \leq \Psi_{c}, \\
0, & \psi_{d}>\Psi_{c},\end{cases}
\end{aligned}
$$

where $A$ is the physical area of the detector in the receiver, which typically is a photodiode $(\mathrm{PD}), T_{s}(\psi)$ is the gain of optical filter which is set as unity in this paper, $g\left(\psi_{d}\right)$ is the gain of an optical concentrator, and $\Psi_{c}$ denotes the FOV angle of mobile terminal. The gain of optical concentrator $g\left(\psi_{d}\right)$ is given as [18]

$$
g\left(\psi_{d}\right)= \begin{cases}\frac{n^{2}}{\sin ^{2}\left(\Psi_{c}\right)}, & 0 \leq \psi_{d} \leq \Psi_{c} \\ 0, & \psi_{d}>\Psi_{c}\end{cases}
$$

where $n$ is the refractive index of optical concentrator. By using similar consideration, the channel DC gain on the first reflection is [20]

$$
d H_{\text {ref }}(0)= \begin{cases}\frac{(m+1) A}{2 \pi^{2} D_{1}^{2} D_{2}^{2}} \rho d A_{\text {wall }} \cos ^{m} \phi_{r} \cos \psi_{r} \cos \alpha \cos \beta T_{s}\left(\psi_{r}\right) g\left(\psi_{r}\right), & 0 \leq \psi_{r} \leq \Psi_{c} \\ 0, & \psi_{r}>\Psi_{c}\end{cases}
$$

The total received power $P_{r}$ is the total power received from directed path $H(0)$ and first reflected path $d H_{\text {ref }}(0)$ which can be written as

$$
P_{r}=\sum_{i}\left\{P_{t} H(0)+\int_{\text {wall }} P_{t} d H_{\text {ref }}(0)\right\},
$$

where the summation is done over the index of LEDs $i$ th. In this work, only two LEDs are considered. However, more LEDs can be treated within this scheme.

Generally, SNR determines the quality of the communication link in the sense that the higher SNR leads to better bit error rate (BER) performance. In VLC, ISI generated by optical path difference has main influenced to SNR. Basically, to treat ISI properly, a sophisticated process is needed [21].
For the sake of simplicity, we consider ISI as noise by adding its power to the noise power and assume the noise model as additive white Gaussian noise model. The on-off keying (OOK) modulation scheme is assumed with rectangular transmitted pulses of duration equal to the bit period $T$. The equation of SNR can be expressed as [19]

$$
\mathrm{SNR}=\frac{\gamma^{2} P_{r \text { Signal }}^{2}}{\gamma^{2} P_{r \text { ISI }}^{2}+\sigma_{\text {noise }}^{2}}
$$

where $\gamma$ is detector responsivity, $\sigma_{\text {noise }}^{2}$ is the noise variance, and $P_{r \text { Signal }}$ and $P_{r \text { ISI }}$ are the optical powers of signal and ISI, respectively. Considering the multipath case with ISI, the 


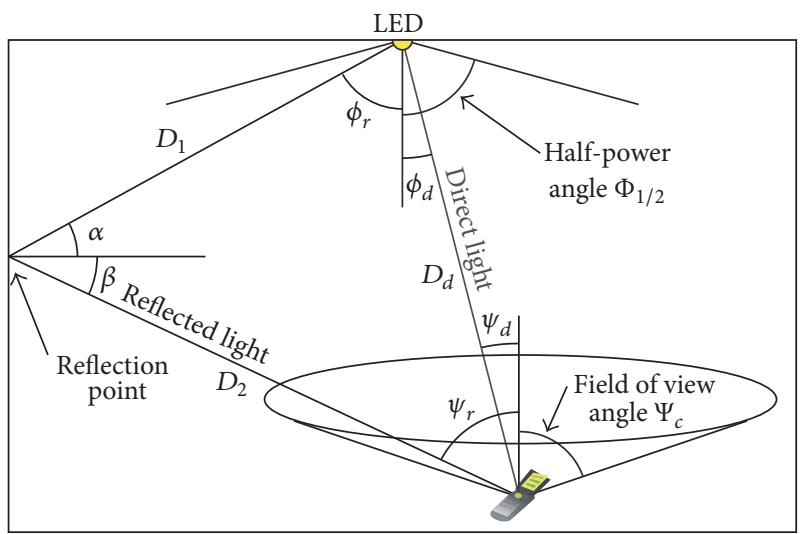

FIGURE 2: Propagation link with first reflection of a VLC system.

TABLE 2: System parameters.

\begin{tabular}{lc}
\hline Parameters and symbols & Values (unit) \\
\hline Bit period $T$ & $10(\mathrm{~ns})$ \\
Gain of optical filter $T_{s}(\psi)$ & 1.0 \\
Photodiode responsibility $\rho$ & $0.54(\mathrm{~A} / \mathrm{W})$ \\
Refractive index of optical concentrator $n$ & 1.5 \\
Detector area in PD $A$ & $1.0\left(\mathrm{~cm}^{2}\right)$ \\
Constant related to noise $C_{1}$ & $1.696 \times 10^{-11}\left(\mathrm{~A}^{2} / \mathrm{W}\right)$ \\
Constant related to noise $C_{2}$ & $1.336 \times 10^{-13}\left(\mathrm{~A}^{2}\right)$ \\
\hline
\end{tabular}

received power $P_{r}$ from (6) can be calculated as the sum of the received power for the desired signal and ISI as [19]

$$
\begin{aligned}
P_{r \text { Signal }} & =\sum_{i} \int_{0}^{T} h_{i}(t) \otimes X(t) d t, \\
P_{r \mathrm{ISI}} & =\sum_{i} \int_{T}^{\infty} h_{i}(t) \otimes X(t) d t,
\end{aligned}
$$

where $h_{i}(t)$ is the impulse response of the $i$ th LED and $X(t)$ is transmitted optical pulse.

According to (7)-(8), more reflection from walls and opaque objects in VLC leads to increasing the ISI level. In case of ISI, where the pulse is broadening at the receiver, the transmitted symbol is more difficult to be demodulated correctly. Consequently, in the presence of reflection and ISI, the BER might be increased rapidly.

The noise variance originates from shot noise and thermal noises and it can be written in a simple form as

$$
\sigma_{\text {noise }}^{2}=C_{1}\left(P_{r \text { Signal }}+P_{r \text { ISI }}\right)+C_{2},
$$

where $C_{1}$ and $C_{2}$ are constants depending mainly on the properties of receiver specification. For our simulation, we adapt numerical values for calculating $C_{1}$ and $C_{2}$ from [19]. Above VLC system parameters are listed in Table 2.

In order to quantify our typical scenario, the calculations of illumination profile, received power, and SNR distribution are performed as shown in Figure 3. According to the results, it is obvious that this LED distribution gives the illumination level satisfied the ISO standard (illumination between 300 and $1500 \mathrm{~lx}$ in all the places in the room). The illumination is highest at the area below each LED and it becomes lower moving towards the corners. Referring to Figure $3(\mathrm{~b})$, the received power is -1 to $4 \mathrm{dBm}$ in most of the places in the room. It is easy to see the abrupt change of the received power at the edge of the view overlap area where MT received lights from both LEDs. Obviously, this overlap area will be changed when the FOV of MT is changed (by simply tilting the receiver). According to the simulation result shown in Figure 3(c), SNR is highest at small areas nearby the wall including four corners where there is less or even no interference. In the view overlap area, SNR is decreased significantly despite the fact that the receivers receive more optical power from different light sources. This is because the total received power in the view overlap area is now the summation of the desired signal power and the ISI power. Moreover, the increasing of ISI power causes the decrease of the link quality in this area by reducing the SNR and increasing the BER. Remark that in the middle line of the room where the directed paths from two LEDs are equal the SNR level is at moderate level as shown in Figure 3(c).

\section{Connectivity and Link Switching}

3.1. VLC Connectivity. In terms of lighting function, the halfpower angle $\Phi_{1 / 2}$ of the LED is an essential parameter in determining the coverage area and the uniformity illuminance. In general, this angle should stay wide for covering throughout the workspace and normally be a fixed parameter corresponding to the type of the LED. The coverage area of an LED can still be changed by varying the receiver plane. The field of view angle $\Psi_{c}$ of the receiver must also be considered in terms of the communication function which is related to connectivity. This angle is defined as the angle of receivable signal rays from LED to receiver. There will be two possibilities that are $\Psi_{c} \leq \Phi_{1 / 2}$ or $\Psi_{c}>\Phi_{1 / 2}$. It has been figured out that reducing $\Psi_{c}$ allows transmitting higher data transmission rate and gives better performance since optical gain is increased (see (4)) and ISI is decreased. Therefore, in this paper, we consider only the case that $\Psi_{c} \leq \Phi_{1 / 2}$. Obviously, in this case, the area on which the receiver can receive the signal is smaller than the coverage area made by the LED and that area is called the view area of the receiver which is given by $A_{\text {view_area }}=\pi\left(h \tan \left(\Psi_{c}\right)\right)^{2}$. In addition, the overlap area at the point view of the receiver which is made by two adjacent view areas is also smaller than the overlap area made by the LEDs and will be called as view overlap area, $A_{\mathrm{vo}}$.

Connectivity is established when the received power at the PDs is greater than the receiver sensitivity (about $-36 \mathrm{dBm}$ ) as reported in previous researches $[9,10]$. However, as shown in Figure 3(b), the received power profile is ranging from about -1 to $4 \mathrm{dBm}$ in most of the places in a typical indoor system. Consequently, connectivity in this paper is defined as the covering percentage of the light with respect to the view area defined by the FOV angle of the PDs. For all lighting configuration, it has been shown that full connectivity at which there is no blind spot throughout the room is desired. To receive full connectivity in conjunction 


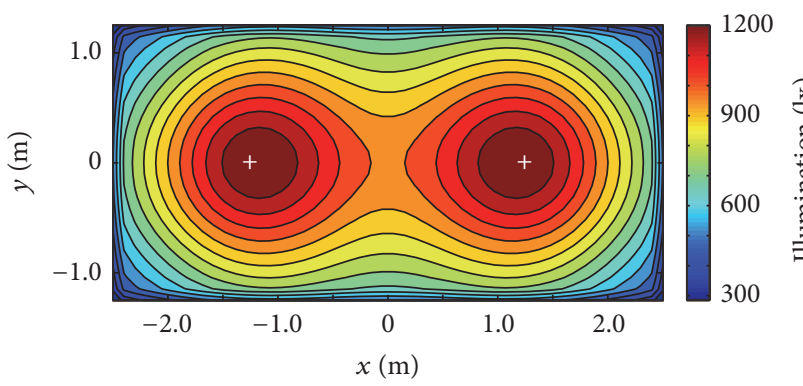

(a)

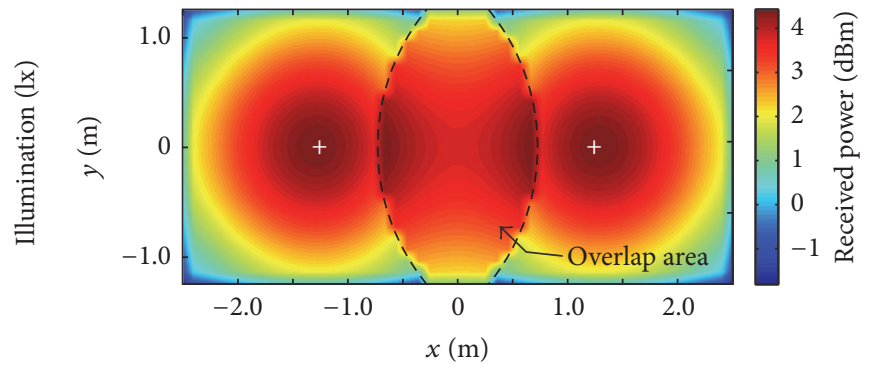

(b)

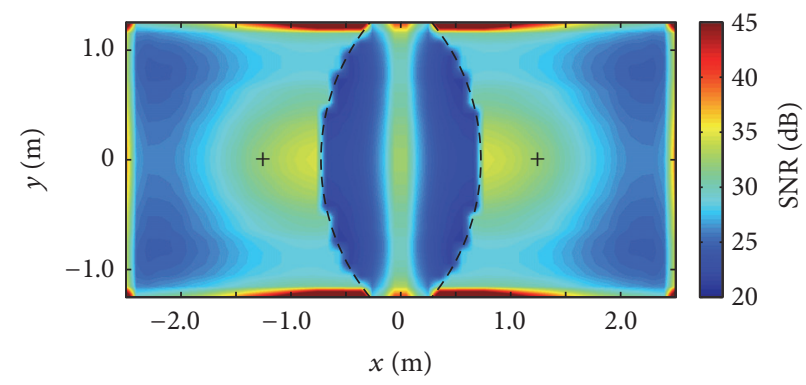

(c)

FIGURE 3: The profile of (a) illumination, (b) receiver power, and (c) SNR. The two LEDs are symmetrically arranged in a horizontal plane. LED1 and LED2 are at $(-1.25,0,2.5)$ and $(1.25,0,2.5)$. The receiver plane is at $z_{0}=0.85 \mathrm{~m}$.

with uniformity, the distance between LEDs must be taken into account; either the number of LEDs used or FOV has to be increased in order to fulfill the condition. From the above discussions, it is clear that too small FOV can lead to the rapid shrinking of MT view area whereby the blind spots occur in the workspace. However, there is a limitation by which increasing the FOV results in high ISI level. In this paper, the blind spot is defined in the plane of receiver not on the floor because receiver is rarely expected to lay on the floor. For the distance between LEDs, either too far or too near distance can lead to the occurrence of blind spots. The relation between this distance and FOV angle with respect to full connectivity under varying position of LEDs will be discussed along with the results from numerical simulations in the next section.

3.2. Link Switching Algorithm. Link switching in VLC is understood as the "handover process" in radio wireless communication and is considered as an indispensable process to maintain and improve the communication link in the scenario of mobility or interference in multiple LED VLC system. The work in [22] focused on optimizing the coverage area of the LED to achieve high average user net rate by taking into account the time spent for handover process. In other words, the research aimed at optimizing the half power angle of the LEDs. In this work, consideration is at both the transmitter and the receiver sides; however, it is assumed that the coverage area of the LED is fixed. Although the communication coverage of a cell in indoor VLC system is normally small and can be varied easily depending on the distance between LEDs, FOV angle, and the receiver plane, yet the speed of MT is normally slow as compared with cellular wireless communication. Consequently, VLC system requires a fast and flexible link switching algorithm [15]. A strong candidate could be the algorithm of relative received power with hysteresis since this algorithm can decrease the amount of link switching and Ping-Pong rate [23]. Referring to this algorithm, when the MT moves from the coverage area of LED1 to the LED2, in the view overlap area $d_{\mathrm{OA}}$, the link switching process is initiated if the received power of the LED2 exceeds the received power of LED1 at least by hysteresis margin $H$ level (in $\mathrm{dB}$ ) which is shown in

$$
10 \log \left(\frac{P_{2}}{P_{1}}\right) \geq H,
$$

where $P_{1}$ and $P_{2}$ represent the received power from the LED1 and LED2, respectively. For the sake of simplicity, $P_{1}$ and $P_{2}$ are assumed to be LOS received power and can be calculated from (3) as

$$
\begin{aligned}
& P_{1}=P_{t} \frac{(m+1) A}{2 \pi D^{2}}\left(\cos ^{m+1} \phi\right) \frac{n^{2}}{\sin ^{2} \Psi_{c}}, \\
& P_{2}=P_{t} \frac{(m+1) A}{2 \pi D^{\prime 2}}\left(\cos ^{m+1} \varphi\right) \frac{n^{2}}{\sin ^{2} \Psi_{c}^{\prime}} .
\end{aligned}
$$

The value of $H$ has been shown to be a critical selection factor for the link switching performances. It affects the amount of link switching and the view overlap area where link switching process occurred. If $H$ is high, the amount of unnecessary link switching is decreased. Yet it requires large view overlap area in order to have successful link switching [24]. This paper will analyze the relation between hysteresis margin $H(\mathrm{~dB})$ and the view overlap area $A_{\mathrm{vo}}$ in respect of the success of link switching process under varying the distance between LED1 and LED2. 


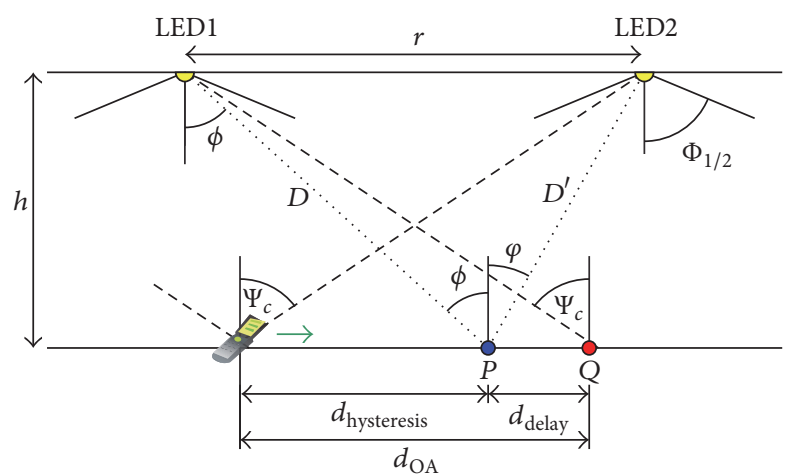

FIgURE 4: The view overlap distance of two LEDs.

We assume MT moves along the straight line which is connected by projection of two LEDs. By this way, MT can move the largest distance in the view overlap area, called view overlap distance, $d_{\mathrm{OA}}$, as shown in Figure 4 . It is assumed that MT can recognize and distinguish the signal powers from LED1 and LED2. When MT moves into the view overlap area, it continuously calculates the signal strengths from two LEDs. At point $P$, it is assumed that $(10)$ is reached, meaning that the link switching is initiated at point $P$. From that point, the MT needs an additional distance in order to get successful link switching. This distance depends on the link switching delay time $T_{d}$ and the speed of MT $v$ as shown in

$$
d_{\text {delay }}=T_{d} \times v .
$$

Substituting (11) into (10) we get

$$
\frac{D^{2} \cos ^{m+1}(\varphi)}{D^{\prime 2} \cos ^{m+1}(\phi)} \geq 10^{H / 10}
$$

Based on (13), after some geometry consideration (see Figure 4 ), the condition of the view overlap distance $d_{\mathrm{OA}}$ can be obtained as

$$
\begin{aligned}
d_{\mathrm{OA}} \geq & h \tan \left(\Psi_{c}\right) \\
& -\sqrt{\frac{h^{2}+\left(h \tan \left(\Psi_{c}\right)-T_{d} \times v\right)^{2}}{(m+3) / 2} \sqrt{10^{H / 10}}-h^{2}}+T_{d} \times v .
\end{aligned}
$$

The condition above is valid if and only if

$$
\frac{h^{2}+\left(h \tan \left(\Psi_{c}\right)-T_{d} \times v\right)^{2}}{\sqrt[(m+3) / 2]{10^{H / 10}}}-h^{2} \geq 0 .
$$

The relation among three parameters, hysteresis margin $H$ $(\mathrm{dB})$, view overlap distance $d_{\mathrm{OA}}$, and the FOV of MT, are shown clearly in (14) and (15).

From (14), one can transform from view overlap distance, $d_{\mathrm{OA}}$, to the distance between LEDs $r$ by using the geometrical relation in Figure 4:

$$
\begin{aligned}
r \leq & h \tan \left(\Psi_{c}\right)+\sqrt{\frac{h^{2}+\left(h \tan \left(\Psi_{c}\right)-T_{d} \times v\right)^{2}}{\sqrt[(m+3) / 2]{10^{H / 10}}-h^{2}}} \\
& -T_{d} \times v .
\end{aligned}
$$

TABLE 3: Additional system parameters.

\begin{tabular}{lc}
\hline Parameter and symbol & Value (unit) \\
\hline Link switching delay time $T_{d}$ & $0.15(\mathrm{~s})$ \\
Speed of MT $v$ & $1(\mathrm{~m} / \mathrm{s})$ \\
Hysteresis margin $H$ & $3-12(\mathrm{~dB})$ \\
Field of view angle of mobile terminal $\Psi_{c}$ & $48-60^{\circ}$ \\
\hline
\end{tabular}

For configuration method, those relations are used to determine the minimum required view overlap distance and the maximum distance between LEDs in order to support link switching process. For system with given distance between LEDs, (14) can be used to determine a suitable hysteresis margin.

\section{Numerical Results}

To demonstrate different lighting layouts, the distance between LED1 and LED2 is varied. At first, LED1 and LED2 are placed in the center of the ceiling and then moved out evenly towards the walls with step of $0.2 \mathrm{~m}$ along length of the room. The full-connectivity requirement is checked first for each lighting layout; then all those lighting layouts satisfying connectivity condition will be calculated for average received power and received average SNR. At last, the relation between lighting layout and hysteresis margin is shown. All above simulations will be implemented for different FOV angle $\Psi_{c}$ with condition $\Psi_{c} \leq \Phi_{1 / 2}$. All the parameters used in the simulations are given in Tables 1 and 2 in conjunction with Table 3.

4.1. Link Quality and Connectivity. Both high level and small fluctuation of received power and SNR are desirable when designing a VLC system. Yet when demonstrating a system with two LEDs, we expected to only consider the level of those two performances and leave the fluctuation issue for the VLC designers who apply our method to configure their systems. Consequently, we do the simulation on the average received power and SNR. Distance between LED1 and LED2 will be varied from $0.2 \mathrm{~m}$ to $4.8 \mathrm{~m}$ with step of $0.2 \mathrm{~m}$ since two LEDs should not be tilted at the same place in the center or right at the edge of the room. For each step, the connectivity and link quality will be calculated at set FOV angle. Figure 5 shows the average received power and average SNR level corresponding to the lighting layouts that give full-connectivity under set FOV angles. Referring to our simulation shown in Figure 5, when FOV angle $\Psi_{c}$ is smaller than $48^{\circ}$ there is no lighting layout providing full-connectivity at all; in other words, the blind area exists at any lighting layout when FOV angle is smaller than $48^{\circ}$. At $\Psi_{c}=48^{\circ}$, the distance between LEDs should only be between 2.4 and $2.6 \mathrm{~m}$ for full-connectivity. With increasing $\Psi_{c}$, the possibility of choosing the lighting layouts that satisfying full-connectivity is also increased; that is, at FOV $50^{\circ}$ the distance can be between 2 and $3 \mathrm{~m}$, and at FOV $55^{\circ}$ distance of 1.5 to $4 \mathrm{~m}$ is acceptable. When FOV reaches $58^{\circ}$ almost the distance between LED1 and LED2 gives full-connectivity. 

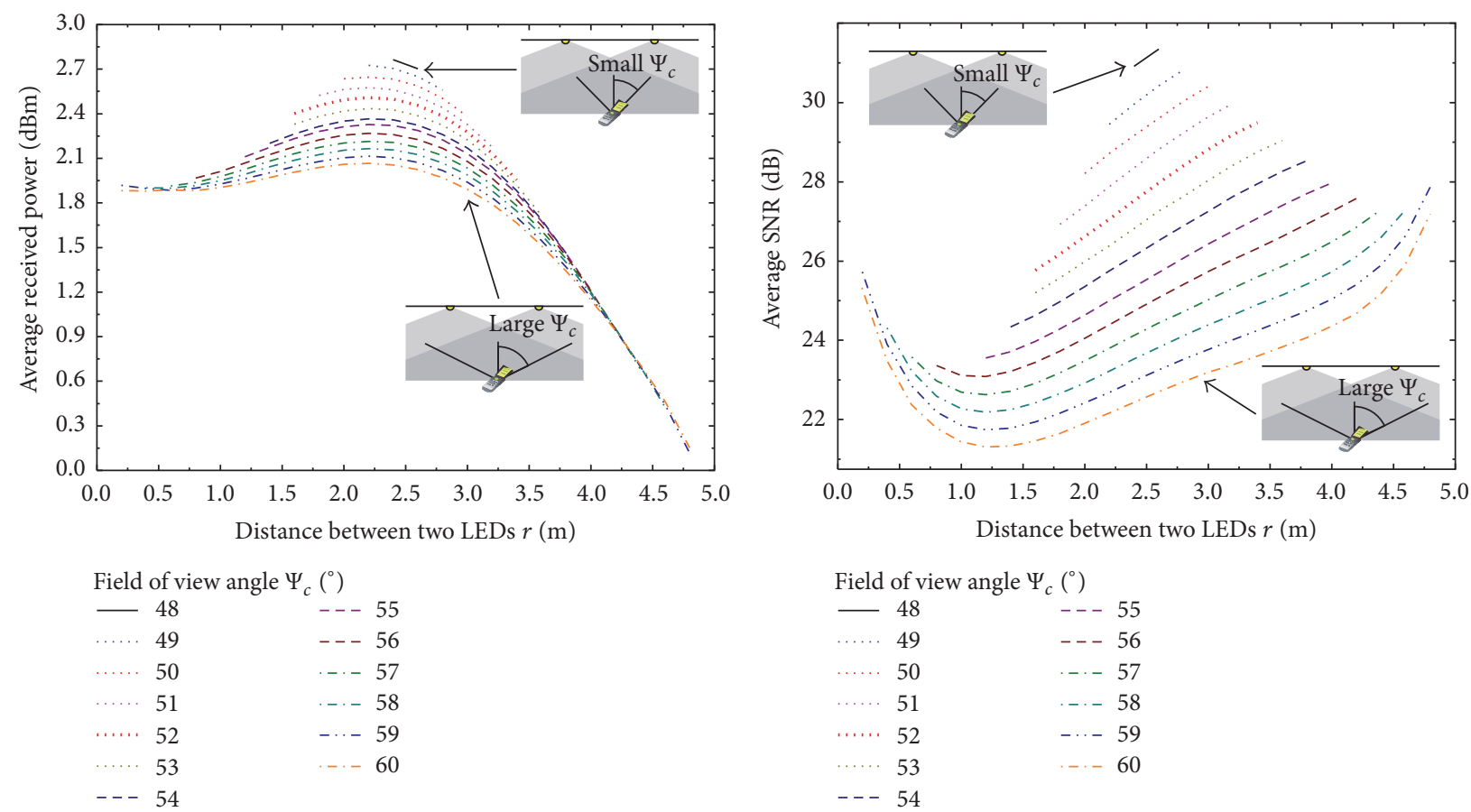

(a)

(b)

FIGURE 5: (a) Average received power and (b) average SNR level corresponding to the lighting layouts that give full-connectivity under different FOV angles.

Referring to Figure 5, for all the layouts that give fullconnectivity, the average received power and average SNR at FOV $48^{\circ}$ are the highest and descending as FOV increased. At FOV $48^{\circ}$, the average received power is about $2.8 \mathrm{dBm}$ and average SNR is about $31 \mathrm{~dB}$. This is due to the fact that when FOV increases the gain of optical concentrator at the detector decreases and it reduces received power afterwards. This reduction in received power together with increasing ISI leads to decreasing SNR level. When two LEDs are installed far from each other and nearby the edge of the room, the average receiver power is very small around $0.15 \mathrm{dBm}$. Furthermore, the trade-off between average received power and average SNR is also easily observed through Figure 5. When SNR is increased, the received power will be decreased and vice versa. This is true especially when the distance between LEDs is large and FOV is small. At large FOV, there will be some places of 2 LEDs that give high average received power and average SNR as well. FOV angle larger than $60^{\circ}$ displays the same trend in our simulation (not shown). As for any communication system, both high level of received power and SNR are desirable. Consequently, in terms of link quality and connectivity, FOV of $48^{\circ}$ and distance between LEDs around $2.5 \mathrm{~m}$ are recommended since this layout gives maximized received power and SNR as well.

4.2. Link Switching with Various Hysteresis Margins. In this section, we simulate the requirement of the view overlap distance between two LEDs for supporting link switching as foregoing discussions to demonstrate the requirement in distance between two LEDs. Those relations are shown in (14)-(16), yet they are inequality formula. We simulate here the equality of those relations. Figure 6 displays the minimum view overlap distance, maximum distance between two LEDs, and FOV at different hysteresis margin for supporting link switching. Referring to Figure 6(a), there is a well satisfaction with the aforementioned theory; that is, when hysteresis margin $H$ increases, the required view overlap distance is also increased. Moreover, the minimum FOV angle also increases at higher $H$. At hysteresis margin $H=3 \mathrm{~dB}$ FOV should be larger than $38^{\circ}$, because smaller FOV angle results in no layout that can satisfy the link switching conditions (see (15)). Similarly, when $H$ equals 6,9 , or $12 \mathrm{~dB}$, the minimum required FOV angles are $50^{\circ}, 56^{\circ}$, and $63^{\circ}$, respectively. For any hysteresis margin, the minimum overlap distance stays quite stable as FOV increase. When $H$ equals $3 \mathrm{~dB}$, the minimum overlap distance is around $1.2 \mathrm{~m}$ for all FOV larger than $38^{\circ}$ and around $1.6 \mathrm{~m}, 2.4 \mathrm{~m}$, and $3.1 \mathrm{~m}$ for $H=6$, 9, and $12 \mathrm{~dB}$, respectively.

Figure 6(b) displays the maximum distance between two LEDs that can well satisfy the link switching condition at different hysteresis margin. From this figure, it is seen that, at all the hysteresis margin values, the increasing of FOV angle leads to an increase of maximum distance between LEDs. At the same FOV, high hysteresis margin often requires small maximum distance between two LEDs. At the FOV of $48^{\circ}$ and the distance between two LEDs $r=2.5 \mathrm{~m}$, link switching process can be applied for hysteresis margin of $3 \mathrm{~dB}$. 


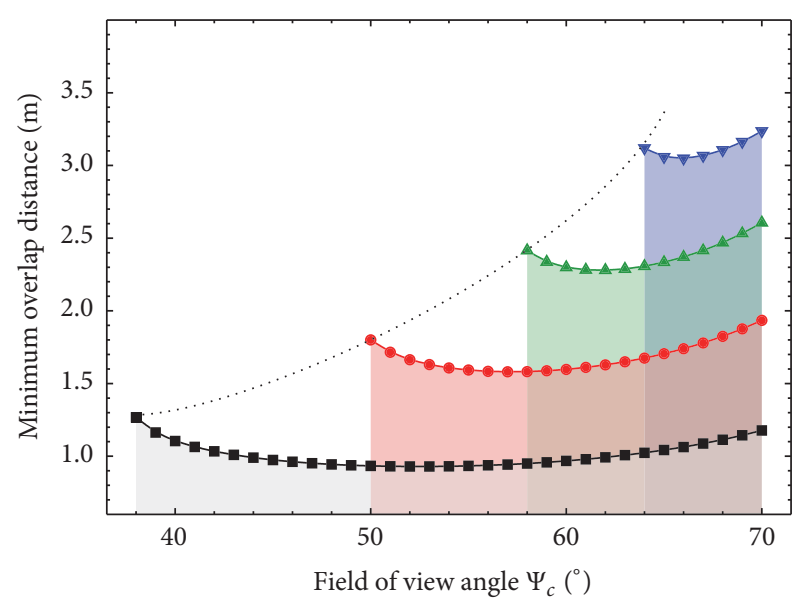

Hysteresis margin $H$

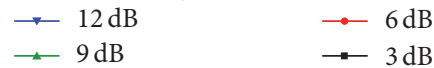

(a)

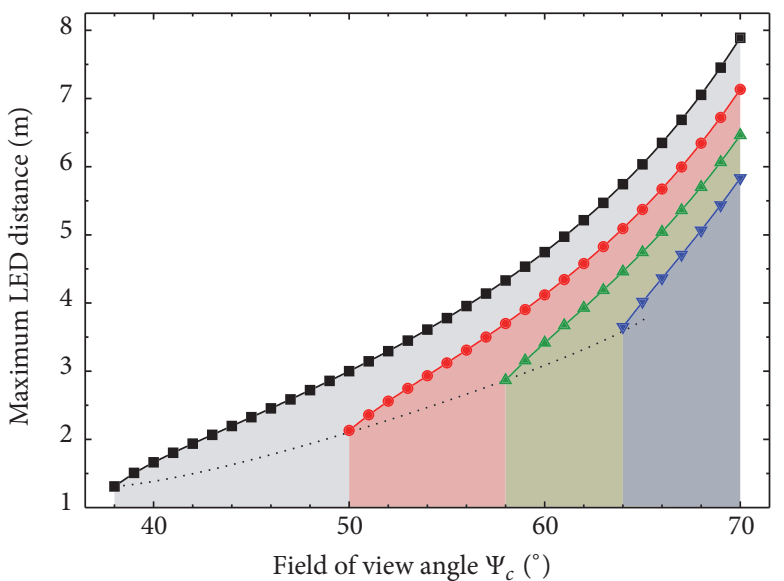

Hysteresis margin $H$

$$
\begin{array}{ll}
\rightarrow 3 \mathrm{~dB} & \rightarrow 9 \mathrm{~dB} \\
-6 \mathrm{~dB} & \rightarrow 12 \mathrm{~dB}
\end{array}
$$

(b)

FIGURE 6: (a) Minimum overlap distance versus FOV and (b) maximum distance between 2 LEDs versus FOV at various hysteresis margin $H$ for supporting link switching.

\section{Conclusion}

We discussed a comprehensive lighting configuration based on the illumination and communication aspects to fully integrate VLC system in indoor environment. It is important to consider the connectivity and link switching performance of an indoor VLC system in order to ensure high quality communication connection. Relationship of various parameters in VLC system, that is, the distance between LEDs, FOV angle of receiver, view area, view overlap area, and view overlap distance in respect of link quality, connectivity, and link switching process, is considered. Based on our simulation approaches, to achieve highest link quality, optimumconnectivity, and fully supporting link switching process the suitable distance between two LEDs at specific FOV angle can be obtained. This work enhances the development of any practical VLC system and can be used to design effective MIMO VLC systems.

\section{Competing Interests}

The authors declare that there is no conflict of interests regarding the publication of this paper.

\section{Acknowledgments}

This work was supported by Naresuan University and VNU University of Engineering and Technology (UET).

\section{References}

[1] D. K. Borah, A. C. Boucouvalas, C. C. Davis, S. Hranilovic, and K. Yiannopoulos, "A review of communication-oriented optical wireless systems," Eurasip Journal on Wireless Communications and Networking, vol. 91, pp. 1-28, 2012.
[2] D. Karunatilaka, F. Zafar, V. Kalavally, and R. Parthiban, "LED based indoor visible light communications: state of the art," IEEE Communications Surveys and Tutorials, vol. 17, no. 3, pp. 1649-1678, 2015.

[3] P. H. Pathak, X. Feng, P. Hu, and P. Mohapatra, "Visible light communication, networking, and sensing: a survey, potential and challenges," IEEE Communications Surveys \& Tutorials, vol. 17, no. 4, pp. 2047-2077, 2015.

[4] D. Wu, C. Chen, Z. Ghassemlooy, and W. Zhong, "Short-range visible light ranging and detecting system using illumination light emitting diodes," IET Optoelectronics, vol. 10, no. 3, pp. 9499, 2016.

[5] J. Vucic, C. Kottke, S. Nerreter, K.-D. Langer, and J. W. Walewski, "513 Mbit/s visible light communications link based on DMTmodulation of a white LED," Journal of Lightwave Technology, vol. 28, no. 24, Article ID 5608481, pp. 3512-3518, 2010.

[6] J. H. Choi, S. W. Koo, and J. Y. Kim, "Influence of optical path difference on visible light communication systems," in Proceedings of the 9th International Symposium on Communications and Information Technology (ISCIT '09), pp. 1247-1251, Incheon, Korea, September 2009.

[7] T.-H. Do and M. S. Yoo, "Received power and SNR optimization for visible light communication system," in Proceedings of the 4th International Conference on Ubiquitous and Future Networks (ICUFN '12), pp. 6-7, Phuket, Thailand, July 2012.

[8] T.-H. Do and M. Yoo, "Optimization for link quality and power consumption of visible light communication system," Photonic Network Communications, vol. 27, no. 3, pp. 99-105, 2014.

[9] A. Burton, H. Le Minh, Z. Ghasemlooy, and S. Rajbhandari, "A study of LED lumination uniformity with mobility for visible light communications," in Proceedings of the International Workshop on Optical Wireless Communications (IWOW '12), Pisa, Italy, October 2012.

[10] A. Burton, Z. Ghassemlooy, S. Rajbhandari, and S.-K. Liaw, "Design and analysis of an angular-segmented full-mobility visible light communications receiver," Transactions on Emerging 
Telecommunications Technologies, vol. 25, no. 6, pp. 591-599, 2014.

[11] IEEE, "IEEE standard for local and metropolitan area networks. Part 15.7: short-range wireless optical communication using visible light," IEEE Standard 802.15.7, 2011.

[12] F. Wang, Z. Wang, C. Qian, L. Dai, and Z. Yang, "Efficient vertical handover scheme for heterogeneous VLC-RF systems," IEEE/OSA Journal of Optical Communications and Networking, vol. 7, no. 12, pp. 1172-1180, 2015.

[13] S. Liang, H. Tian, B. Fan, and R. Bai, "A novel vertical handover algorithm in a hybrid visible light communication and LTE system," in Proceedings of the IEEE 85th Vehicular Technology Conference (VTC Fall '16), pp. 1-5, Montréal, Canada, January 2016.

[14] D. Wu, Z. Ghassemlooy, W.-D. Zhong, and C. Chen, "Cellular indoor OWC systems with an optimal lambertian order and a handover algorithm," in Proceedings of the 7th International Symposium on Telecommunications (IST '14), pp. 777-782, IEEE, Tehran, Iran, September 2014.

[15] T. Nguyen, M. Z. Chowdhury, and Y. M. Jang, "A novel link switching scheme using pre-scanning and RSS prediction in visible light communication networks," Eurasip Journal on Wireless Communications and Networking, vol. 2013, article 293, 2013.

[16] P. F. Mmbaga, J. Thompson, and H. Haas, "Performance analysis of indoor diffuse VLC MIMO channels using angular diversity detectors," Journal of Lightwave Technology, vol. 34, no. 4, pp. 1254-1266, 2016.

[17] R. S. Berns, Billmeyer and Saltzman's Principles of Color Technology, John Wiley \& Sons, New York, NY, USA, 2000.

[18] J. R. Barry, Wireless Infrared Communications, Kluwer Academic Press, Boston, Mass, USA, 1994.

[19] T. Komine and M. Nakagawa, "Fundamental analysis for visiblelight communication system using LED lights," IEEE Transactions on Consumer Electronics, vol. 50, no. 1, pp. 100-107, 2004.

[20] F. R. Gfeller and U. Bapst, "Wireless in-house data communication via diffuse infrared radiation," Proceedings of the IEEE, vol. 67, no. 11, pp. 1474-1486, 1979.

[21] W. Hauk, F. Bross, and M. Ottka, "The calculation of error rates for optical fiber systems," IEEE Transactions on Communications, vol. 26, no. 7, pp. 1119-1126, 1978.

[22] S. Pergoloni, M. Biagi, S. Colonnese, R. Cusani, and G. Scarano, "Optimized LEDs footprinting for indoor visible light communication networks," IEEE Photonics Technology Letters, vol. 28, no. 4, pp. 532-535, 2016.

[23] S. Moghaddam, V. Tabataba, and A. Falahati, "New handoff initiation algorithm (optimum combination of hysteresis and threshold based methods)," in Proceedings of the 52nd Vehicular Technology Conference (IEEE-VTS Fall '00), pp. 1567-1574, Boston, Mass, USA, September 2000.

[24] S. Lal and D. K. Panwar, "Coverage analysis of handoff algorithm with adaptive hysteresis margin," in Proceedings of the International Conference on Information Technology (ICIT '07), December 2007. 

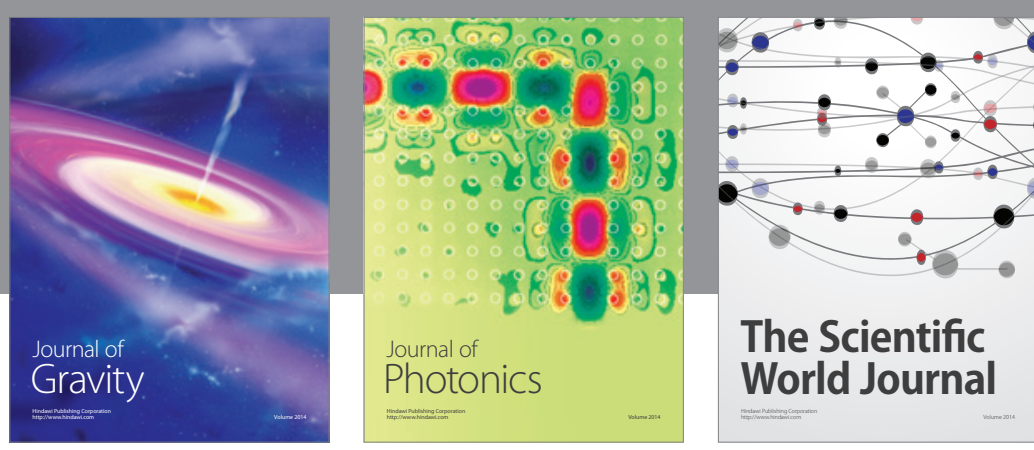

The Scientific World Journal
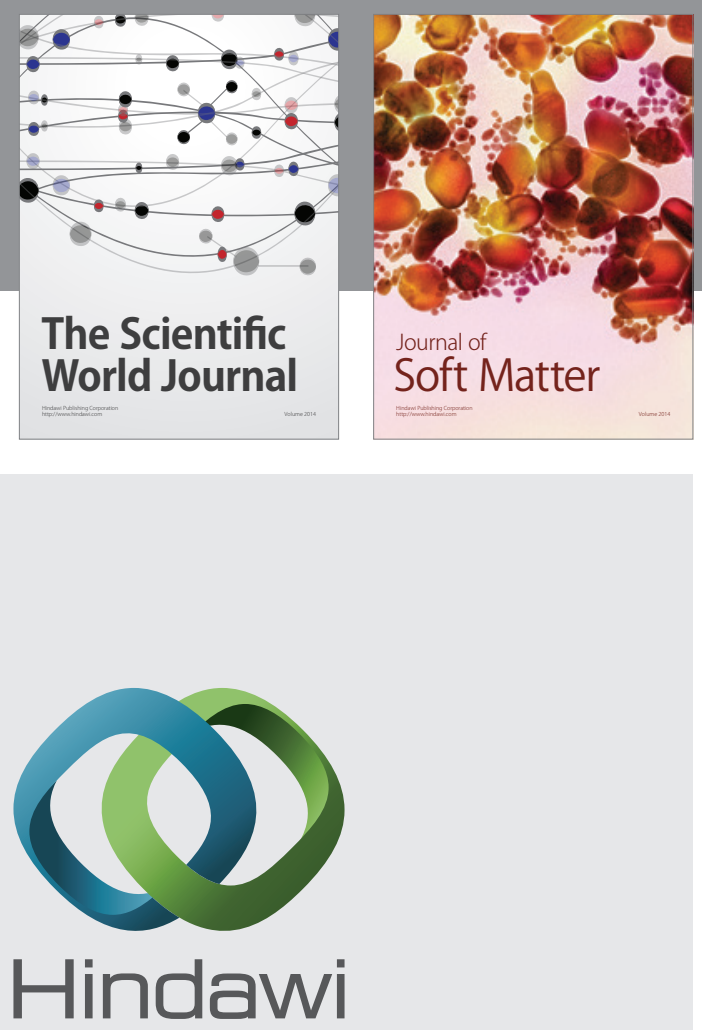

Submit your manuscripts at

http://www.hindawi.com

nternational Journal of

Statistical Mechanics
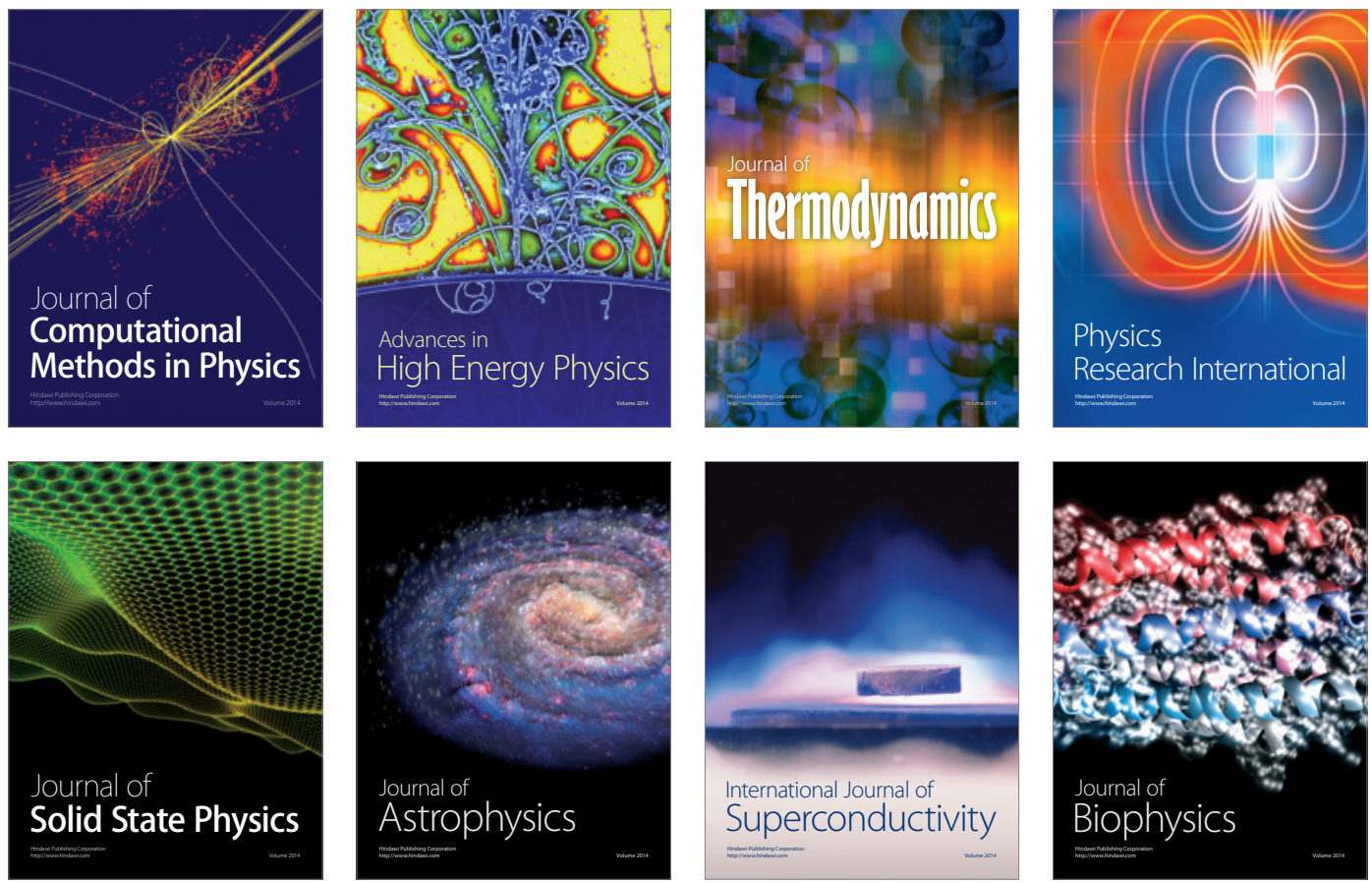
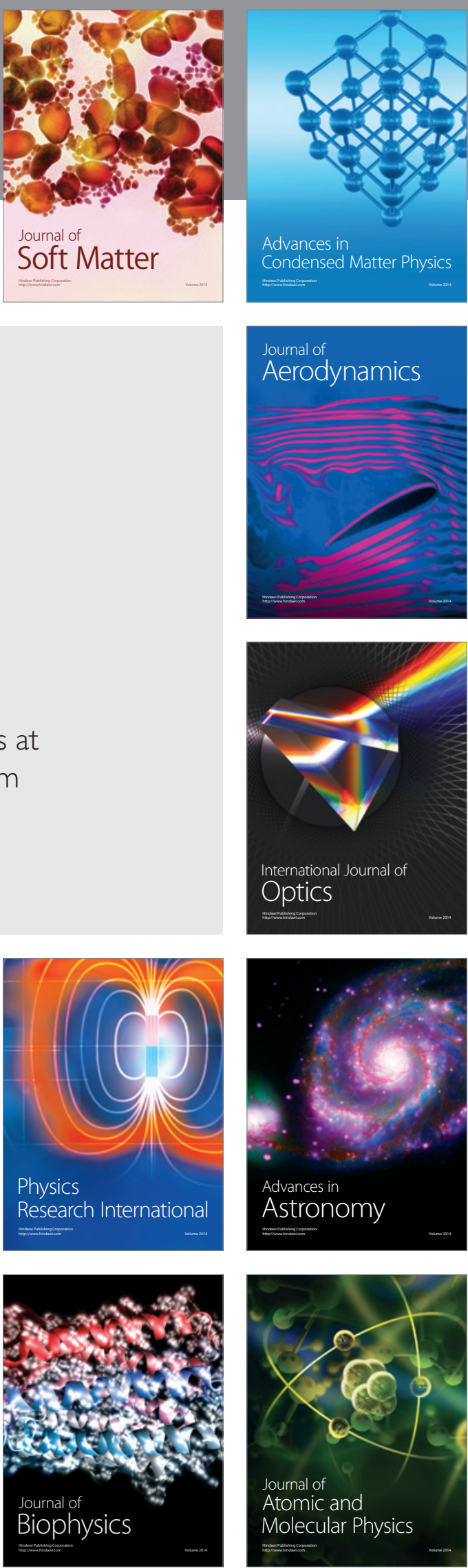